\title{
Platelet/lymphocyte ratio was associated with impaired myocardial perfusion and both in-hospital and long-term adverse outcome in patients with ST-segment elevation acute myocardial infarction undergoing primary coronary intervention
}

\author{
Cuneyt Toprak ${ }^{1}$, Mehmet M. Tabakci ${ }^{1}$, Zeki Simsek ${ }^{1}$, Ugur Arslantas ${ }^{1}$, Halil I. Durmus ${ }^{2}$, Lutfi Ocal ${ }^{1}$, \\ Muhittin Demirel $^{1}$, Burak Ozturkeri ${ }^{1}$, Ender Ozal ${ }^{3}$, Ramazan Kargin ${ }^{1}$ \\ ${ }^{1}$ Kosuyolu Kartal Heart Training and Research Hospital, Istanbul, Turkey \\ 2Department of Cardiology, Tavsanli Doc. Dr. Mustafa Kalemli State Hospital, Kutahya, Turkey \\ ${ }^{3}$ Cardiology Clinic, Pendik State Hospital, Istanbul, Turkey
}

Postep Kardiol Inter 2015; 11, 4 (42): 288-297

DOI: $10.5114 /$ pwki.2015.55599

\begin{abstract}
A bstract
Introduction: Platelet/lymphocyte ratio (PLR) has been shown to be an inflammatory and thrombotic biomarker for coronary heart disease, but its prognostic value in ST-segment elevation myocardial infarction (STEMI) has not been fully investigated.

Aim: To investigate the relationship between PLR and no-reflow, along with the in-hospital and long-term outcomes in patients with STEMI.

Material and methods: In the present study, we included 304 consecutive patients suffering from STEMI who underwent primary percutaneous coronary intervention $(\mathrm{p}-\mathrm{PCI})$. Patients were stratified according to PLR tertiles based on the blood samples obtained in the emergency room upon admission. No-reflow after p-PCI was defined as a coronary thrombolysis in myocardial infarction (TIMI) flow grade $\leq 2$ after vessel recanalization, or TIMI flow grade 3 together with a final myocardial blush grade (MBG) $<2$.

Results: The mean follow-up period was 24 months (range: 22-26 months). The number of patients characterized with no-reflow was counted to depict increments throughout successive PLR tertiles ( $14 \%$ vs. $20 \%$ vs. $45 \%, p<0.001)$. In-hospital major adverse cardiovascular events and death increased as the PLR increased $(p<0.001, p<0.001)$. Long-term MACE and death also increased as the PLR increased $(p<0.001, p<0.001)$. Multivariable logistic regression analysis revealed that PLR remained an independent predictor for both in-hospital $(\mathrm{OR}=1.01,95 \% \mathrm{Cl}: 1.00-1.01 ; p=0.002)$ and major long-term $(\mathrm{OR}=1.01,95 \% \mathrm{Cl}: 1.00-1.01$; $p<0.001)$ adverse cardiac events.

Conclusions: Platelet/lymphocyte ratio on admission is a strong and independent predictor of both the no-reflow phenomenon and long-term prognosis following $\mathrm{p}-\mathrm{PCl}$ in patients with STEMI.
\end{abstract}

Key words: platelet/lymphocyte ratio, in-hospital mortality, long-term mortality, no-reflow, ST-segment elevation acute myocardial infarction.

\section{Introduction}

It is well known that angiographic no-reflow is associated with short- and long-term morbidity and mortality in acute ST-segment elevation myocardial infarction (STEMI) [1, 2]. Although the pathophysiology of no-reflow has not been fully elucidated, its etiology is likely multifactorial and complex. Inflammatory processes and excess thrombotic activity play an important role in angio- graphic no-reflow $[3,4]$. The identification of predictors of these processes, which has important implications for assessing patient management, improving clinical outcomes and for the design of future trials, is intended to improve myocardial recovery. The platelet to lymphocyte ratio (PLR) was introduced as a potential marker to determine excess thrombotic activity [5] and inflammation in oncologic and cardiac disorders [6, 7].

\section{Corresponding author:}

Cuneyt Toprak PhD, Kosuyolu Kartal Heart Training and Research Hospital, Denizer St. no: 2, Cevizli/Kartal, 34864 Istanbul, Turkey, phone: +90 2165001500, e-mail: cuneytoprak@hotmail.com

Received: 3.02.2015, accepted: 11.08.2015. 
Recently, PLR has been shown to be an independent predictor of coronary no-reflow and in-hospital mortality in patients with STEMI who underwent primary percutaneous coronary intervention (p-PCI) [8-10]. However, data regarding the association of PLR levels with longterm mortality in the setting of STEMI are lacking.

\section{Aim}

The primary aim of this study was to evaluate the association of PLR with angiographic coronary blood flow as well as to evaluate the in-hospital and long-term outcomes in patients with STEMI undergoing $\mathrm{p}-\mathrm{PCl}$.

\section{Material and methods}

\section{Patient population}

The initial study population was composed of 408 consecutive patients diagnosed definitively with STEMI who underwent $\mathrm{p}-\mathrm{PCl}$. Among these, 104 patients were excluded because of not receiving $\mathrm{p}-\mathrm{PCl}(n=14)$, missing laboratory values $(n=44)$ or lacking clinical follow-up data $(n=56)$. Finally the study population consisted of 304 patients. Based on the PLR upon admission, patients were stratified into tertiles ( $1^{\text {st }}$ tertile: $<141,2^{\text {nd }}$ tertile: 141-217 and $3^{\text {rd }}$ tertile: $\left.>217\right)$. The inclusion criteria were as follows: (a) presentation within the first $12 \mathrm{~h}$ of onset of chest pain (18 $\mathrm{h}$ for cardiogenic shock), (b) ST elevation of at least $1 \mathrm{~mm}$ in two or more contiguous leads (2 mm for leads V1-V3), or new-onset left bundle branch block. Exclusion criteria included treatment with thrombolytic drugs in the previous $24 \mathrm{~h}$, active infections, recent major surgical procedure or trauma, previously proved systemic inflammatory disease, known malignancy, and end-stage liver and renal failures. Written informed consent was obtained from all patients. The study was conducted in accordance with the Declaration of Helsinki, and the study protocol was approved by the local ethics committee.

\section{Angiographic analysis}

All $\mathrm{p}-\mathrm{PCl}$ procedures were performed using the standard femoral approach with a 7-Fr guiding catheter. Pharmacological treatment before $\mathrm{p}-\mathrm{PCl}$ included $300 \mathrm{mg}$ of chewable aspirin, a loading dose of $600 \mathrm{mg}$ of clopidogrel and an intravenous bolus of unfractionated heparin at a dose of $70 \mathrm{U} / \mathrm{kg}$ of body weight. The use of glycoprotein Ilb/IIla receptor blockers (tirofiban) with $10 \mathrm{mg} / \mathrm{kg}$ bolus and $0.15 \mathrm{mg} / \mathrm{kg} / \mathrm{min}$ intravenous infusion was left to the primary operator's discretion. Direct stenting was performed whenever possible; in the remaining cases, balloon predilatation was performed. Choice of stents (bare-metal or drug-eluting stent) was left to the operator's discretion. To achieve maximal dilation, an intracoronary injection of nitroglycerin $(100 \mu \mathrm{g})$ preceded each coronary angiogram.
All patients were treated with maintenance doses of clopidogrel (75 mg once daily for 12 months) and aspirin (300 mg on admission and then $100 \mathrm{mg}$ indefinitely).

Assessment of angiograms obtained at baseline angiography was performed by two independent and experienced interventional cardiologists who were blinded to all data; final agreement was made for $95 \%$ of discordances, which were resolved by consensus. We primarily calculated thrombolysis in myocardial infarction (TIMI) thrombus grade based on the initial diagnostic angiogram. In TIMI thrombus grade 0 , no cine-angiographic characteristics of thrombus are present; in TIMI thrombus grade 1 , possible thrombus is present with such angiographic characteristics as decreased contrast density, haziness, irregular lesion contour, or a smooth convex "meniscus" at the site of total occlusion suggestive but not diagnostic of thrombus; in TIMI thrombus grade 2, there is definite thrombus, with the largest dimensions $\leq 1 / 2$ the vessel diameter; in TIMI thrombus grade 3 , there is definite thrombus but with the largest linear dimension $\geq 1 / 2$ but $\leq 2$ vessel diameters; in TIMI thrombus grade 4 , there is definite thrombus, with the largest dimension 2 vessel diameters; and in TIMI thrombus grade 5, there is total occlusion. Then, after restoring antegrade flow through the guidewire or small balloon predilatation in patients with TIMI thrombus grade 5; coronary angiogram enabled restratification of the underlying residual thrombus (final TIMI thrombus grade) [4, 11]. We then stratified the final TIMI thrombus grades as low thrombus burden or high thrombus burden, based on scores 0 to 3 or 4 to 5 , respectively. No-reflow after $\mathrm{p}-\mathrm{PCl}$ was defined as a coronary TIMI flow grade $\leq 2$ after vessel recanalization or TIMI flow grade 3 together with a final myocardial blush grade (MBG) < 2 (group 1), and angiographic success was defined as TIMI 3 flow together with a final $M B G \geq 2$ (group 2), as previously described [12].

\section{Laboratory tests and echocardiography}

An antecubital venous blood sample was drawn upon admission from each patient either in the coronary care unit or in the emergency department before administration of any medication. Complete blood counts, which included platelets, total white blood cells, neutrophils and lymphocytes, were obtained using an automatic blood counter (Beckman Coulter LH 750, Fullerton, CA). Neutrophil/lymphocyte ratio (NLR) and PLR were calculated as the ratio of the neutrophils and platelets to lymphocytes, both obtained from the same automated blood samples which were taken at admission before the p-PCI. C-reactive protein (CRP) levels, which were measured using a BN II model Nephelometer (Dade Behring, Marburg, Germany), were also entered into the database. Other biochemical parameters including lipid profiles were determined by virtue of commercially available methods and kits. Transthoracic echocardiographic studies were 
performed using a S5-1 transducer on an iE33 ultrasound machine (Philips Medical Systems, Andover, MA, USA). Left ventricular ejection fraction (LVEF) was calculated from 4- and 2-chamber views using the modified Simpson biplane method.

\section{Clinical follow-up}

Clinical follow-up data regarding participating patients were obtained either through out-patient examination or by telephone contact 24 months (interquartile range: 22-26 months) after $\mathrm{p}-\mathrm{PCl}$.

Major adverse cardiovascular events (MACE) comprised a composite of death, non-fatal re-infarction, and target vessel revascularization (TVR) during the in-hospital or long-term follow-up period. The definition of re-infarction was made according to the Third Universal Definition of Myocardial Infarction [13]. Target vessel revascularization was defined as needed for $\mathrm{PCl}$ or coronary surgery because of restenosis or reocclusion of the infarct-related artery.

\section{Statistical analysis}

Statistical analysis was performed using SPSS for Windows (version 19.0; SPSS Inc, Chicago, Illinois). Continuous variables were expressed as mean \pm standard deviation or median (interquartile range) depending on normality, assessed using the Kolmogorov-Smirnov test. Among tertiles, measurements were compared using a one-way ANOVA model test followed by Tukey's post hoc test. Each categorical variable was expressed as number and percentage of patients. Group means for continuous variables were compared using either the unpaired Student's $t$ test or the Mann-Whitney $U$ test according to normality.

Categorical variables were compared using the chisquare test or Fisher's exact test, as appropriate. The association of different variables with in-hospital and longterm MACE was calculated in univariate analysis. Multiple logistic regression analysis was performed to eliminate variables. Variables showing marginal associations with no-reflow on univariate testing were included in the regression analysis $(p<0.1)$. The stepwise method with backward elimination was used. Odds ratios with $95 \%$ confidence intervals were calculated. A receiver-operating characteristic (ROC) curve was constructed to determine the sensitivity and specificity of PLR and the optimal cut-off value for predicting long-term MACE in patients with STEMI. The Kaplan-Meier method was used to construct the cumulative survival curve for long-term cardiovascular mortality, and the log-rank test was used to assess the differences. A $p$ value of $<0.05$ was considered statistically significant.

\section{Results}

Table I shows the baseline characteristics of patients according to PLR tertiles. There was no statistically sig- nificant difference between the PLR tertiles with regard to the baseline characteristics (age, gender, risk factors, etc.) and pain-to-balloon time. However, fasting glucose and serum CRP levels were observed to increase across the subsequent PLR tertiles. Hemoglobin levels and LVEF values, on the other hand, were getting poorer and poorer across the tertile groups (Table I). Platelet levels were significantly higher (249.8 $\pm 65.1,255.9 \pm 73.1,302.7 \pm 107.8$, $p<0.001$ for trend across tertiles), while lymphocyte levels were significantly lower $(2.6 \pm 1.4,1.4 \pm 0.4,0.9 \pm 0.3$, $p<0.001$ for trend across tertiles). Neutrophil/lymphocyte ratio increased as the PLR increased (105.6 \pm 26.9 , $175.2 \pm 22.4,352.4 \pm 158.7, p<0.001$ for trend across tertiles). In addition, patients presented with cardiogenic shock significantly higher in tertile $3(p=0.02)$.

A higher prevalence of no-reflow after $\mathrm{p}-\mathrm{PCl}$ was observed as the PLR tertiles increased ( $p<0.001$ for trend across tertiles). As the tertile increased, the prevalence of multivessel disease increased $(p=0.010)$. Final TIMI thrombus grades of the study population consisted of low thrombus burden, significantly higher in tertile 1 compared to tertile 3 , and high thrombus burden, significantly lower in tertile 1 compared to tertile 3 (Table II).

We established a multivariable logistic regression model by using no-reflow as the dependent variable with adjustments for significant variables (as identified from the univariable regression analysis - PLR and NLR on admission, fasting glucose, previous coronary artery disease (CAD), CRP, glycoprotein Ilb/IIla antagonist, diabetes mellitus (DM), aspirin usage, $\beta$-blocker usage, cardiogenic shock and final TIMI thrombus grades), ending up with PLR on admission (odds ratio (OR) 1.01, 95\% confidence interval $(\mathrm{Cl})$ : $1.00-1.01 ; p=0.003)$ as the independent predictor for the no-reflow phenomenon (Table III). During the in-hospital period, the incidence of MACE was significantly higher in patients with no-reflow (26 (66.7\%) vs. $13(33.3 \%), p<0.001)$, and mortality was higher in the no-reflow group $(p<0.001)$. The number of patients who died during follow-up was significantly higher in the no-reflow group compared to the patients who survived $(p<0.001)$. Also, in the long-term follow-up, MACE incidence was significantly higher in patients with no-reflow (43 (62\%) vs. 26 (38\%), $p<0.001)$.

The associations of different variables with the in-hospital and long-term MACE and mortality were evaluated in univariate analysis. In univariate analysis, variables with a $p$ value $<0.05$ were analyzed using a multivariate logistic regression model. For in-hospital MACE, PLR, NLR, CRP, $\beta$-blocker, DM, previous CAD, hospitalization, final TIMI thrombus grades, no-reflow, cardiogenic shock, and LVEF were analyzed using a multivariate logistic regression model. The PLR was an independent predictor of in-hospital MACE (OR $=1.01,95 \% \mathrm{Cl}: 1.00-$ $1.01 ; p=0.007$ ) (Table IV) after multivariate analysis. For long-term MACE, PLR, NLR, CRP, $\beta$-blocker, aspirin, fasting 
Table I. Baseline characteristics

\begin{tabular}{|c|c|c|c|c|}
\hline \multirow[t]{2}{*}{ Variable } & \multicolumn{3}{|c|}{ Platelet/lymphocyte } & \multirow[t]{2}{*}{ Value of $p$} \\
\hline & Tertile $1(<141)(n=101)$ & Tertile $2(141-217)(n=102)$ & Tertile $3(>217)(n=101)$ & \\
\hline Age [years] & $60.0 \pm 9.5$ & $60.0 \pm 11.2$ & $59.4 \pm 9.6$ & 0.883 \\
\hline Men & $83(82)$ & $86(84)$ & 77 (76) & 0.317 \\
\hline Body mass index [kg/m²] & $26.3 \pm 4.1$ & $26.5 \pm 4.6$ & $26.7 \pm 4.4$ & 0.824 \\
\hline Hypertension & $42(42)$ & $44(43)$ & $49(48)$ & 0.582 \\
\hline Diabetes & $26(26)$ & $22(22)$ & $27(27)$ & 0.663 \\
\hline Current smoking & $58(57)$ & $52(51)$ & $58(57)$ & 0.566 \\
\hline Previous CAD & $13(13)$ & $14(14)$ & $22(22)$ & 0.164 \\
\hline Peak CK-MB [U/I] & $189.6 \pm 122.2$ & $181.3 \pm 110.6$ & $194.5 \pm 105.5$ & 0.704 \\
\hline C-reactive protein [mg/l] & $5.3 \pm 6.2$ & $7.1 \pm 6.5$ & $8.1 \pm 6.2$ & 0.009 \\
\hline Creatinine [mg/dl] & $0.88 \pm 0.23$ & $0.90 \pm 0.24$ & $0.92 \pm 0.23$ & 0.546 \\
\hline LVEF (\%) & $50.0 \pm 9.3$ & $49.9 \pm 9.3$ & $47.0 \pm 10.6$ & 0.053 \\
\hline Fasting glucose [mg/dl] & $144.5 \pm 69.5$ & $161.7 \pm 94.6$ & $187.6 \pm 119.2$ & 0.007 \\
\hline LDL cholesterol [mg/dl] & $121.6 \pm 320$ & $128.4 \pm 34.4$ & $122.6 \pm 31.2$ & 0.288 \\
\hline HDL cholesterol [mg/dl] & $39.5 \pm 10.6$ & $37.6 \pm 9.9$ & $39.7 \pm 10.0$ & 0.285 \\
\hline Triglyceride [mg/dl] & $125.9 \pm 49.6$ & $136.0 \pm 65.3$ & $121.9 \pm 48.6$ & 0.175 \\
\hline Total cholesterol [mg/dl] & $189.0 \pm 38.1$ & $197.8 \pm 38.7$ & $188.9 \pm 36.8$ & 0.161 \\
\hline Hemoglobin [g/dl] & $13.9 \pm 1.6$ & $13.5 \pm 1.6$ & $12.7 \pm 1.9$ & $<0.001$ \\
\hline Platelets $\left[\times 10^{3} \mu \mathrm{l}\right]$ & $249.8 \pm 65.1$ & $255.9 \pm 73.1$ & $302.7 \pm 107.8$ & $<0.001$ \\
\hline$W B C\left[\times 10^{3} \mu \mathrm{l}\right]$ & $13.5 \pm 5.7$ & $12.7 \pm 4.2$ & $12.6 \pm 4.2$ & 0.313 \\
\hline Lymphocytes $\left[\times 10^{3} \mu \mathrm{l}\right]$ & $2.6 \pm 1.4$ & $1.4 \pm 0.4$ & $0.9 \pm 0.3$ & $<0.001$ \\
\hline Neutrophil/lymphocyte ratio & $4.3 \pm 2.4$ & $7.4 \pm 3.2$ & $13.1 \pm 7.1$ & $<0.001$ \\
\hline Platelet/lymphocyte ratio & $105.6 \pm 26.9$ & $175.2 \pm 22.4$ & $352.4 \pm 158.7$ & $<0.001$ \\
\hline Mean platelet volume [fl] & $8.4 \pm 1.1$ & $8.6 \pm 1.3$ & $8.6 \pm 1.3$ & 0.480 \\
\hline Glycoprotein IIb/IIla antagonist & $37(37)$ & $36(35)$ & $42(42)$ & 0.623 \\
\hline Pain-to-balloon time [h] & $2.4 \pm 0.8$ & $2.6 \pm 0.9$ & $2.6 \pm 0.9$ & 0.459 \\
\hline Cardiogenic shock & $0(0)$ & $3(3)$ & $7(7)$ & 0.021 \\
\hline Hospitalization [days] & $6.3 \pm 1.7$ & $6.6 \pm 1.9$ & $6.7 \pm 1.6$ & 0.335 \\
\hline \multicolumn{5}{|l|}{ Previous medications: } \\
\hline Aspirin & $17(17)$ & $15(15)$ & $29(29)$ & 0.027 \\
\hline ACE inhibitors/ARB & $36(36)$ & $35(34)$ & $38(38)$ & 0.885 \\
\hline$\beta$-Blocker & $30(30)$ & $21(21)$ & $31(31)$ & 0.202 \\
\hline Statin & $24(24)$ & $23(23)$ & $19(19)$ & 0.673 \\
\hline
\end{tabular}

Results presented as mean $\pm S D$ or $n(\%)$. CAD - Coronary artery disease, CK-MB - creatine kinase-MB, LDL-low-density lipoprotein, HDL - high-density lipoprotein, LVEF - left ventricle ejection fraction, TIMI - thrombolysis in myocardial infarction, ACE - angiotensin-converting enzyme, ARB - angiotensin receptor blocker, WBC - white blood cell count. 
Table II. Clinical outcomes, angiographic and procedural characteristics

\begin{tabular}{|c|c|c|c|c|}
\hline \multirow[t]{2}{*}{ Variable } & \multicolumn{3}{|c|}{ Platelet/lymphocyte ratio } & \multirow[t]{2}{*}{ Value of $p$} \\
\hline & Tertile $1(<141)(n=101)$ & Tertile $2(141-217)(n=102)$ & Tertile $3(>217)(n=101)$ & \\
\hline Infarct-related coronary artery: & & & & 0.010 \\
\hline Left anterior descending & $46(45)$ & $48(47)$ & $60(59)$ & \\
\hline Circumflex & $20(20)$ & $18(18)$ & $11(11)$ & \\
\hline Right & $35(35)$ & $36(35)$ & $30(30)$ & \\
\hline Final TIMI flow grade: & & & & 0.001 \\
\hline 0 & $2(2)$ & $1(1)$ & $4(4)$ & \\
\hline 1 & $1(1)$ & $1(1)$ & $8(8)$ & \\
\hline 2 & $8(8)$ & $15(15)$ & $23(23)$ & \\
\hline 3 & $90(89)$ & $85(83)$ & $66(65)$ & \\
\hline Final TIMI thrombus grade scale: & & & & $<0.001$ \\
\hline Low thrombus burden & $67(66)$ & $74(73)$ & $45(45)$ & \\
\hline High thrombus burden & $34(34)$ & $28(27)$ & $56(55)$ & \\
\hline Multivessel disease & $31(31)$ & $48(47)$ & $51(50)$ & 0.010 \\
\hline Primary percutaneous coronary i & tervention: & & & 0.309 \\
\hline Stent implantation & $100(99)$ & $96(94)$ & $96(95)$ & \\
\hline Bare-metal stent & $38(38)$ & $36(35)$ & $30(30)$ & \\
\hline Drug-eluting stent & $62(61)$ & $60(59)$ & $66(65)$ & \\
\hline Stent length [mm] & $23.4 \pm 10.9$ & $23.7 \pm 10.0$ & $23.8 \pm 10.3$ & 0.963 \\
\hline Stent diameter [mm] & $3.18 \pm 0.4$ & $3.25 \pm 0.4$ & $3.23 \pm 0.4$ & 0.341 \\
\hline No-reflow & $14(14)$ & $20(20)$ & $45(45)$ & $<0.001$ \\
\hline \multicolumn{5}{|l|}{ In-hospital: } \\
\hline Deaths & $2(2)$ & $4(4)$ & $14(14)$ & $<0.001$ \\
\hline$\overline{M A C E}$ & $7(7)$ & $10(10)$ & $22(22)$ & $<0.001$ \\
\hline \multicolumn{5}{|l|}{ At 2-year follow-up: } \\
\hline Deaths & $8(8)$ & $10(10)$ & $25(25)$ & $<0.001$ \\
\hline$\overline{M A C E}$ & $11(11)$ & $20(20)$ & $38(38)$ & $<0.001$ \\
\hline
\end{tabular}

Results presented as mean \pm SD or $n(\%)$. MACE - Major adverse cardiovascular events.

glucose, previous CAD, hospitalization, final TIMI thrombus grades, no-reflow, cardiogenic shock, and LVEF were included in the multivariate logistic regression model. At multivariate analysis, PLR was also an independent predictor of long-term MACE (OR = 1.01, 95\% Cl: 1.00-1.01; $p<0.001$ ) (Table V).

For in-hospital mortality, PLR $(\mathrm{OR}=1.01,95 \% \mathrm{Cl}$ : $1.00-1.01 ; p=0.024)$, no-reflow $(\mathrm{OR}=7.57,95 \% \mathrm{Cl}$ : 2.21-25.9; $p=0.001)$, and cardiogenic shock (OR = 20.4, 95\% Cl: 3.98-105; $p<0.001)$ were independent predictors of in-hospital mortality (Table $\mathrm{VI}$ ) after the multivariate analysis. For long-term mortality, PLR $(O R=1.01$,
95\% Cl: $1.00-1.01 ; p=0.005)$, no-reflow (OR = 13.3, $95 \% \mathrm{Cl}: 5.40-32.6 ; p<0.001)$, cardiogenic shock $(\mathrm{OR}=6.18,95 \% \mathrm{Cl}: 1.01-32.6 ; p=0.049)$, and CRP $(\mathrm{OR}=1.10,95 \% \mathrm{Cl}: 1.04-1.17 ; p=0.001)$ were also independent predictors of long-term mortality in multivariate analysis (Table VII).

The ROC curves of PLR for predicting the outcomes are shown in Figure 1. In this study, a PLR level of $>201$, measured upon admission, had $70 \%$ sensitivity and $66 \%$ specificity in predicting the development of long-term adverse cardiac events. The cumulative survival curves for long-term cardiovascular death with different PLR 
Table III. Effects of various variables on no-reflow phenomenon in univariate and multivariate logistic regression analyses

\begin{tabular}{|c|c|c|c|c|c|c|}
\hline \multirow[t]{2}{*}{ Variable } & \multicolumn{3}{|c|}{ Univariate } & \multicolumn{3}{|c|}{ Multivariate } \\
\hline & Value of $p$ & OR & $95 \% \mathrm{Cl}$ & Value of $p$ & OR & $95 \% \mathrm{Cl}$ \\
\hline Fasting glucose & 0.003 & 1.18 & $1.10-1.96$ & & & \\
\hline Previous CAD & $<0.001$ & 4.31 & $2.25-8.15$ & & & \\
\hline Diabetes & 0.002 & 2.45 & $1.40-4.29$ & 0.003 & 2.90 & $1.43-5.86$ \\
\hline Glycoprotein IIb/IIla antagonist & 0.007 & 2.05 & $1.22-3.45$ & & & \\
\hline$\beta$-Blocker & $<0.001$ & 2.79 & $1.61-4.81$ & 0.044 & 2.05 & $1.02-4.11$ \\
\hline Aspirin & $<0.001$ & 5.05 & $2.78-9.17$ & 0.001 & 3.35 & $1.62-6.91$ \\
\hline Cardiogenic shock & 0.002 & 12.6 & $2.60-60.5$ & & & \\
\hline TIMI thrombus grade scale & $<0.001$ & 5.43 & $3.11-9.47$ & $<0.001$ & 3.90 & $2.03-7.47$ \\
\hline C-reactive protein & $<0.001$ & 1.10 & $1.19-1.60$ & 0.018 & 1.06 & $1.01-1.11$ \\
\hline NLR & $<0.001$ & 1.09 & $1.04-1.15$ & & & \\
\hline PLR & $<0.001$ & 1.01 & $1.00-1.01$ & 0.003 & 1.01 & $1.00-1.01$ \\
\hline
\end{tabular}

$N L R$ - Neutrophil/lymphocyte ratio, $P L R$ - platelet/lymphocyte ratio, OR - odds ratio, CI - confidence interval.

Table IV. Effects of multiple variables on in-hospital MACE in univariate and multivariate logistic regression analyses

\begin{tabular}{|c|c|c|c|c|c|c|}
\hline \multirow[t]{2}{*}{ Variable } & \multicolumn{3}{|c|}{ Univariate } & \multicolumn{3}{|c|}{ Multivariate } \\
\hline & Value of $p$ & OR & $95 \% \mathrm{Cl}$ & Value of $p$ & OR & $95 \% \mathrm{Cl}$ \\
\hline Diabetes & 0.082 & 1.87 & $0.91-3.82$ & & & \\
\hline Previous CAD & 0.028 & 2.34 & $1.07-5.10$ & & & \\
\hline LVEF & 0.025 & 0.96 & $0.92-0.99$ & & & \\
\hline Hospitalization & 0.003 & 1.31 & $1.09-1.56$ & & & \\
\hline$\beta$-Blocker & 0.012 & 2.37 & $1.19-4.75$ & & & \\
\hline Cardiogenic shock & $<0.001$ & 33.9 & $6.89-167$ & 0.012 & 8.94 & $1.62-49.3$ \\
\hline TIMI thrombus grade scale & 0.001 & 3.30 & $1.63-6.65$ & & & \\
\hline No-reflow & $<0.001$ & 8.00 & $3.85-16.6$ & 0.001 & 4.16 & $1.84-9.39$ \\
\hline C-reactive protein & $<0.001$ & 1.12 & $1.06-1.17$ & 0.005 & 1.08 & $1.02-1.14$ \\
\hline NLR & $<0.001$ & 1.10 & $1.04-1.60$ & & & \\
\hline PLR & $<0.001$ & 1.01 & $1.00-1.01$ & 0.007 & 1.01 & $1.00-1.01$ \\
\hline
\end{tabular}

tertiles were constructed using the Kaplan-Meier method, and their differences were compared by the log-rank test (Figure 2).

\section{Discussion}

The present study results showed that PLR was an independent predictor of no-reflow in patients with STEMI who underwent $\mathrm{p}-\mathrm{PCl}$. To the best of our knowledge, this is the first study demonstrating the relation of PLR on admission with in-hospital and long-term mortality in patients with STEMI. Prognostic values of red cell distri- bution width (RDW), mean platelet volume (MPV), white blood cells (WBCs) and NLR, which can be derived from the complete blood count, in STEMI have been investigated, but PLR has recently been investigated as a new predictor for major adverse cardiovascular outcomes [7, 8, 14-16].

The no-reflow phenomenon occurs in approximately half of the patients with STEMI after $\mathrm{p}-\mathrm{PCl}$. Previous studies have shown that no-reflow has a strong negative impact on clinical outcome, as it is independently associated with early post-infarct complications, late repeat hospital stays for heart failure, and mortality [1, 2]. Although nu- 
Table V. Effects of various variables on long-term major adverse cardiac events in univariate and multivariate logistic regression analyses

\begin{tabular}{|c|c|c|c|c|c|c|}
\hline \multirow[t]{2}{*}{ Variable } & \multicolumn{3}{|c|}{ Univariate } & \multicolumn{3}{|c|}{ Multivariate } \\
\hline & Value of $p$ & OR & $95 \% \mathrm{Cl}$ & Value of $p$ & OR & $95 \% \mathrm{Cl}$ \\
\hline Fasting glucose & 0.034 & 1.00 & $1.00-1.01$ & & & \\
\hline Previous CAD & 0.029 & 2.07 & $1.06-4.02$ & & & \\
\hline LVEF & 0.047 & 0.97 & $0.94-1.00$ & & & \\
\hline Hospitalization & $<0.001$ & 1.35 & $1.16-1.57$ & 0.008 & 1.30 & $1.07-1.60$ \\
\hline$\beta$-Blocker & 0.012 & 1.93 & $1.09-3.42$ & & & \\
\hline Aspirin & 0.005 & 2.35 & $1.27-4.33$ & & & \\
\hline Cardiogenic shock & 0.001 & 35.1 & $4.36-282$ & & & \\
\hline TIMI thrombus grade scale & $<0.001$ & 3.25 & $1.86-5.67$ & & & \\
\hline No-reflow & $<0.001$ & 9.14 & $5.00-16.7$ & $<0.001$ & 4.82 & $2.45-9.48$ \\
\hline C-reactive protein & $<0.001$ & 1.14 & $1.09-1.19$ & $<0.001$ & 1.13 & $1.07-1.20$ \\
\hline NLR & $<0.001$ & 1.12 & $1.06-1.17$ & & & \\
\hline PLR & $<0.001$ & 1.01 & $1.00-1.01$ & $<0.001$ & 1.01 & $1.00-1.01$ \\
\hline
\end{tabular}

Table VI. Effects of various variables on in-hospital mortality in univariate and multivariate logistic regression analyses

\begin{tabular}{|c|c|c|c|c|c|c|}
\hline \multirow[t]{2}{*}{ Variable } & \multicolumn{3}{|c|}{ Univariate } & \multicolumn{3}{|c|}{ Multivariate } \\
\hline & Value of $p$ & OR & $95 \% \mathrm{Cl}$ & Value of $p$ & OR & $95 \% \mathrm{Cl}$ \\
\hline Fasting glucose & 0.041 & 1.01 & $1.00-1.01$ & & & \\
\hline Cardiogenic shock & $<0.001$ & 50.4 & $11.7-218$ & $<0.001$ & 20.4 & $3.98-105$ \\
\hline TIMI thrombus grade scale & 0.050 & 2.52 & $0.99-6.36$ & & & \\
\hline No-reflow & $<0.001$ & 14.0 & $4.53-43.5$ & 0.001 & 7.57 & $2.21-25.9$ \\
\hline C-reactive protein & 0.001 & 1.10 & $1.04-1.17$ & & & \\
\hline NLR & 0.001 & 1.11 & $1.04-1.17$ & & & \\
\hline PLR & $<0.001$ & 1.01 & $1.00-1.01$ & 0.024 & 1.01 & $1.00-1.01$ \\
\hline
\end{tabular}

merous potential explanations of the development of no-reflow have been put forward, the significance of any single mechanism is not yet fully understood.

Increased PLR can be associated with the no-reflow phenomenon. Platelet-induced inflammatory processes might play a pivotal role in atherothrombosis [17]. Increased platelet counts may reflect underlying inflammation as several inflammatory mediators stimulate megakaryocytic proliferation and produce relative thrombocytosis. Activated platelets release inflammatory and mitogenic substances into the local microenvironment, which would promote the recruitment of more platelets and leukocytes $[17,18]$. The rush of platelets and neutrophils that follows reperfusion may lead to the formation of neutrophil-platelet aggregates that plug the microcirculation and reperfusion-related injury [19]. A positive correlation was found between the acute phase reactants and proinflammatory proteins (CRP, interleukin (IL)-1, IL-6, and tumor necrosis factor $\alpha$ ) and an elevated platelet count in nonspecific inflammatory conditions [20].

In addition, recent studies have shown that patients with CAD have increased platelet and monocyte aggregates in their bloodstream, which was associated with plaque instability, worse in-hospital outcomes, and increased risk of future cardiac events $[21,22]$. On the other hand, elevated numbers of lymphocytes have also been speculated to be related to an increase in plaque stability [23]. Previous studies reported that lymphocytopenia was independently related to mechanical complications and mortality in patients with acute myocardial infarction [24, 25]. 
Table VII. Effects of various variables on long-term mortality in univariate and multivariate logistic regression analyses

\begin{tabular}{|c|c|c|c|c|c|c|}
\hline \multirow[t]{2}{*}{ Variable } & \multicolumn{3}{|c|}{ Univariate } & \multicolumn{3}{|c|}{ Multivariate } \\
\hline & Value of $p$ & OR & $95 \% \mathrm{Cl}$ & Value of $p$ & OR & $95 \% \mathrm{Cl}$ \\
\hline Diabetes & 0.042 & 2.03 & $1.03-4.02$ & & & \\
\hline LVEF & 0.020 & 0.96 & $0.93-0.99$ & & & \\
\hline Hospitalization & 0.006 & 1.27 & $1.07-1.51$ & & & \\
\hline Glycoprotein IIb/IIla antagonist & 0.024 & 2.11 & $1.10-4.05$ & & & \\
\hline Aspirin & 0.030 & 2.20 & $1.08-4.48$ & & & \\
\hline Cardiogenic shock & $<0.001$ & 29.6 & $6.04-145$ & 0.049 & 6.18 & $1.01-37.9$ \\
\hline TIMI thrombus grade scale & $<0.001$ & 4.00 & $2.01-7.96$ & & & \\
\hline No-reflow & $<0.001$ & 21.6 & $9.37-49.7$ & $<0.001$ & 13.2 & $5.40-32.6$ \\
\hline C-reactive protein & $<0.001$ & 1.14 & $1.09-1.20$ & 0.001 & 1.10 & $1.04-1.17$ \\
\hline NLR & $<0.001$ & 1.11 & $1.05-1.17$ & & & \\
\hline PLR & $<0.001$ & 1.01 & $1.00-1.01$ & 0.005 & 1.01 & $1.00-1.01$ \\
\hline
\end{tabular}

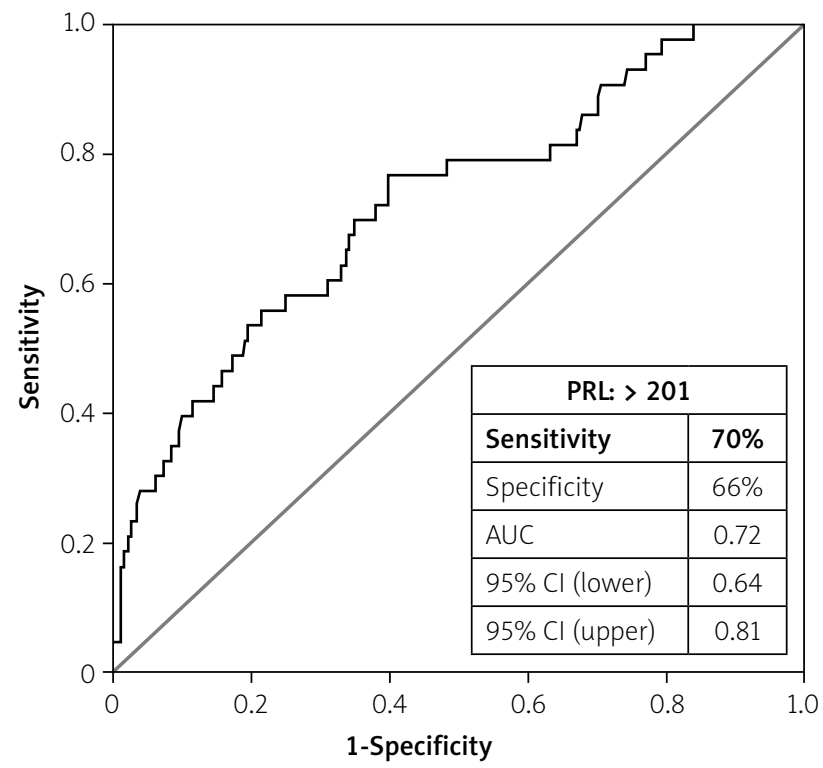

Figure 1. Receiver operating characteristic curve of PLR for predicting long-term MACE

The other underlying mechanism of no-reflow may be related to excess thrombotic status. Intracoronary thrombus and high thrombus burden have an important role in occurrence of no-reflow [4]. In addition, previous studies suggested that treatments such as glycoprotein IIb/IIla inhibitors, or procedures such as thrombectomy, which reduce thrombus burden, improve both epicardial and myocardial perfusion [26]. Release of various mediators such as IL-1 and IL-3 during the proinflammatory state causes megakaryocyte proliferation and an increase in

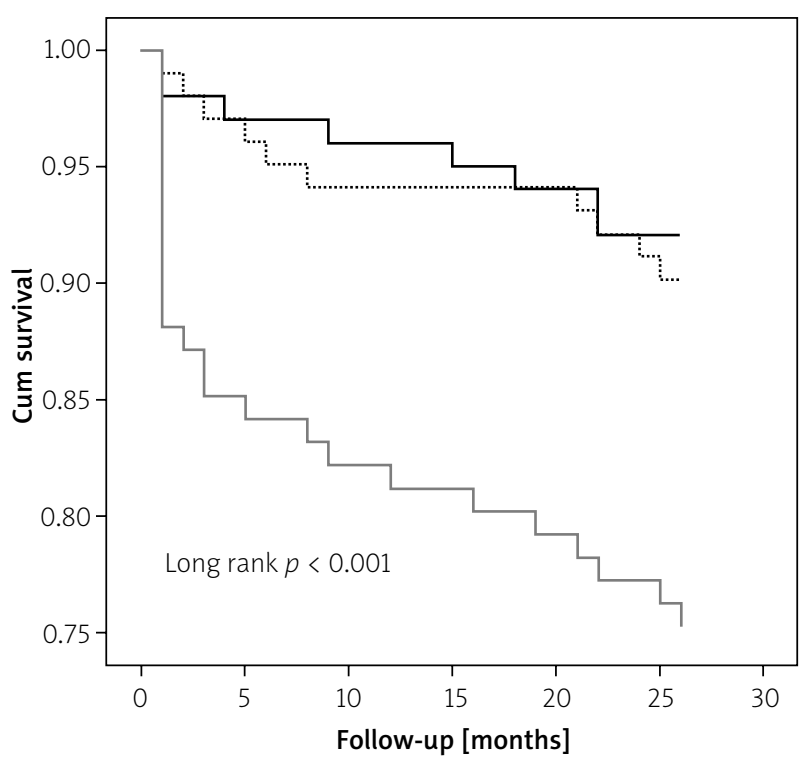

PRL group $\quad \square<141 \quad \ldots: \ldots: 141-217 \quad-\sqcap>217$

Figure 2. Kaplan-Meier curves for long-term survival according to tertiles of PLR in the entire cohort of patients

circulating platelet count $[27,28]$. Thus, increased platelet counts may indicate enhanced thrombocyte activation and a prothrombotic state [29]. Alternatively, PLR is a combined marker of lymphocytopenia and thrombocytosis and may better indicate no-reflow as well as mortality.

In our study, we found that a significant relation between the TIMI thrombus burden scale and PLR as well as high thrombus burden is an independent predictor of no-reflow after p-PCl. Furthermore, Gürsoy et al. re- 
cently reported that increased PLR was an independent predictor for thrombosis in patients with prosthetic valve thrombosis [5]. It was speculated that higher PLR is a marker of excess prothrombotic status, as we were able to support this hypothesis in our study. Therefore, we believe that PLR is not only a biomarker which reflects acute inflammatory activity but also an indicator of the thrombus burden from which more distal culprit lesions clot, causing embolization. Large-scale future studies concerning no-reflow in patient with STEMI may highlight the underlying mechanisms behind the prothrombotic and inflammatory effects of this novel hematologic parameter.

Azab et al. investigated the prognostic value of PLR in non-STEMI, by following patients for 4 years [7]. At follow-up, patients with PLR $\leq 118$ had the lowest allcause mortality rate of $17 \%$, whereas patients with PLR $\geq 176$ had $42 \%$ all-cause mortality. In another study, Temiz et al. found that NLR was not an independent predictor and that $P L R \geq 144$ was a predictor of increased risk for in-hospital mortality in patients with STEMI treated with fibrinolytic therapy [8]. Ugur et al. found that patients with PLR $\geq 175$ had a higher rate of 6-month all-cause deaths as compared with patients with PLR, but they did not find any correlation between PLR and in-hospital as well as one month mortality [30]. Cicek et al. found that a combination of PLR and NLR could identify high-risk patients undergoing $\mathrm{PPCl}$ and predict long-term mortality in STEMI, whereas NLR or PLR alone was not found to be an independent predictor of all-cause mortality [31]. Also, they did not find any significant relation between PLR and MACE. In the present study, 304 patients with STEMI who underwent primary $\mathrm{PCl}$ from a single center registry were followed up to 24 months (interquartile range: 22-26 months). According to our results, high levels of PLR were independently correlated with in-hospital and long-term MACE among patients with STEMI who undergo $\mathrm{p}-\mathrm{PCl}$. Our study has some limitations; it is a single-center study with a small number of patients. However, our study population is a homogeneous group of unselected patients with STEMI undergoing $\mathrm{p}-\mathrm{PCl}$ within $12 \mathrm{~h}$ from symptom onset, which is directly relevant to most patients undergoing $\mathrm{p}-\mathrm{PCl}$ in the general population. There was an independent association between $\beta$-blocker and ASA usage and the no-reflow phenomenon. This incidental finding in contrast to the expectation may be due to the small number of patients and population characteristics such as the high incidence of patients with a history of coronary artery disease in the no-reflow group. The odds ratio of PLR (1.01) for in-hospital and long-term major adverse cardiac events was found to be low, which may seem weak in statistical analysis. The odds ratio of PLR $=1.01$ means that each 1 unit increase in PLR results in a $1 \%$ increase in mortality risk, since PLR values are large numbers (ranging: 16-1267). These results are statistically significant. When we assign patients to high and low PLR groups, statistical analysis results in an OR $=3.16(95 \%$ Cl: 1.56-6.42) for long-term MACE, which means that being in the high PLR group increases the risk of long-term MACE development 3.16-fold.

\section{Conclusions}

The present study results demonstrated that PLR is a simple and readily available biomarker, and is an independent predictor of in-hospital and long-term mortality in patients with STEMI undergoing $\mathrm{p}-\mathrm{PCI}$. It may also enable risk stratification and selection of a treatment strategy in patients with STEMI prior to or during coronary interventional procedures. The prognostic role of this parameter in STEMI and related complications should be investigated in future trials.

\section{Conflict of interest}

The authors declare no conflict of interest.

\section{References}

1. Brosh D, Assali AR, Mager A, et al. Effect of no-reflow during primary percutaneous coronary intervention for acute myocardial infarction on six-month mortality. Am J Cardiol 2007; 99: 442-5.

2. Bolognese L, Carrabba N, Parodi G, et al. Impact of microvascular dysfunction on left ventricular remodeling and long-term clinical outcome after primary coronary angioplasty for acute myocardial infarction. Circulation 2004; 109: 1121-6.

3. Rezkalla SH, Kloner RA. No-reflow phenomenon. Circulation 2002; 105: 656-62.

4. Tanboga IH, Topcu S, Aksakal E, et al. Determinants of angiographic thrombus burden in patients with ST-segment elevation myocardial infarction. Clin Appl Thromb Hemost 2014; 20: 716-22.

5. Gürsoy OM, Karakoyun S, Kalçık M, et al. Usefulness of novel hematologic inflammatory parameters to predict prosthetic mitral valve. Am J Cardiol 2014; 113: 860-4.

6. Kwon HC, Kim SH, Oh SY, et al. Clinical significance of preoperative neutrophil-lymphocyte versus plateletlymphocyte ratio in patients with operable colorectal cancer. Biomarkers 2012; 17: 216-22.

7. Azab B, Shah N, Akerman M, et al. Value of platelet/lymphocyte ratio as a predictor of all-cause mortality after non-ST elevation myocardial infarction. J Thromb Thrombolysis 2012; 34: 326-34.

8. Temiz A, Gazi E, Gungor O, et al. Platelet/lymphocyte ratio and risk in-hospital mortality in patients with ST-elevated myocardial infarction. Med Sci Monit 2014; 22: 660-5.

9. Kurtul A, Yarlioglues M, Murat SN, et al. Usefulness of the platelet-to-lymphocyte ratio in predicting angiographic reflow after primary percutaneous coronary intervention in patients with acute ST-segment elevation myocardial infarction. Am J Cardiol 2014; 114: 342-7.

10. Acet $\mathrm{H}$, Ertaş F, Akıl MA, et al. Relationship between hematologic indices and global registry of acute coronary events risk score in patients with ST-segment elevation myocardial infarction. Clin Appl Thromb Hemost 2014 May 8 [Epub ahead of print]. DOI: 10.1177/1076029614533145.

11. Sianos G, Papafaklis MI, Serruys PW. Angiographic thrombus burden classification in patients with ST-segment elevation 
myocardial infarction treated with percutaneous coronary intervention. J Invasive Cardiol 2010; 22: 6B-14B.

12. Niccoli G, Burzotta F, Galiuto L, Crea F. Myocardial no-reflow in humans. J Am Coll Cardiol 2009; 54: 281-92.

13. Thygesen K, Alpert JS, Jaffe AS, et al. Third universal definition of myocardial infarction. Circulation 2012; 126: 2020-35.

14. Liu Q, Wang T, Chen R, et al. Mean platelet volume predicts left descending artery occlusion in patients with non-ST-elevation myocardial infarction. Platelets 2014; 25: 246-51.

15. Akin F, Kose N, Ayca B, et al. Relation between red cell distribution width and severity of coronary artery disease in patients with acute myocardial infarction. Angiology 2013; 64: 592-6.

16. Kaya MG, Akpek M, Lam YY, et al. Prognostic value of neutrophil/ lymphocyte ratio in patients with ST-elevated myocardial infarction undergoing primary coronary intervention: a prospective, multicenter study. Int J Cardiol 2013; 168: 1154-9.

17. Gawaz M, Langer H, May AE. Platelets in inflammation and atherogenesis. J Clin Invest 2005; 115: 3378 -84.

18. Peerschke El, Yin W, Ghebrehiwet B. Complement activation on platelets: implications for vascular inflammation and thrombosis. Mol Immunol 2010; 47: 2170-5.

19. Mehta JL, Nichols WW, Mehta P. Neutrophils as potential participants in acute myocardial ischemia: relevance to reperfusion. J Am Coll Cardiol 1988; 11: 1309-16.

20. Alexandrakis MG, Passam FH, Moschandrea IA, et al. Levels of serum cytokines and acute phase proteins in patients with essential and cancer-related thrombocytosis. Am J Clin Oncol 2003; 26: 135-40.

21. Furman MI, Benoit SE, Barnard MR, et al. Increased platelet reactivity and circulating monocyte-platelet aggregates in patients with stable coronary artery disease. J Am Coll Cardiol 1998; 31: 352-8.

22. Zhang SZ, Jin YP, Qin GM, et al. Association of platelet-monocyte aggregates with platelet activation, systemic inflammation, and myocardial injury in patients with non-ST elevation acute coronary syndromes. Clin Cardiol 2007; 30: 26-31.

23. Zazula AD, Precoma-Neto D, Gomes AM, et al. An assessment of neutrophils/lymphocytes ratio in patients suspected of acute coronary syndrome. Arq Bras Cardiol 2008; 90: 31-6.

24. Widmer A, Linka AZ, Attenhofer Jost $\mathrm{CH}$, et al. Mechanical complications after myocardial infarction reliably predicted using C-reactive protein levels and lymphocytopenia. Cardiology 2003; 99: 25-31.

25. Dragu R, Khoury S, Huri S, et al. Predictive value of white blood cell subtypes for long-term outcome following myocardial infarction. Atherosclerosis 2008; 196: 405-12.

26. Tarsia G, De Michele M, Polosa D, et al. Manual versus nonmanual thrombectomy in primary and rescue percutaneous coronary angioplasty. Heart Vessels 2010; 25: 275-81.

27. Klinger MH, Jelkmann W. Role of blood platelets in infection and inflammation. J Interferon Cytokine Res 2002; 22: 913-22.

28. Alexandrakis MG, Passam FH, Moschandrea IA, et al. Levels of serum cytokines and acute phase proteins in patients with essential and cancer-related thrombocytosis. Am J Clin Oncol 2003; 26: 135-40.

29. Gary T, Pichler M, Belaj K, et al. Platelet-to-lymphocyte ratio: a novel marker for critical limb ischemia in peripheral arterial occlusive disease patients. PLoS One 2013; 8: e67688.

30. Ugur M, Gul M, Bozbay M, et al. The relationship between platelet to lymphocyte ratio and the clinical outcomes in ST elevation myocardial infarction underwent primary coronary intervention. Blood Coagul Fibrinolysis 2014; 25: 806-11.
31. Cicek G, Acıkgoz SK, Bozbay M, et al. Neutrophil-lymphocyte ratio and platelet-lymphocyte ratio combination can predict prognosis in patients with ST-segment elevation myocardial infarction undergoing primary percutaneous coronary intervention. Angiology 2015; 66: 441-7. 